\title{
Hepatic Steatosis in Chronic Hepatitis B as Measured by Controlled Attenuation Parameter: Frequency and Distribution
}

\author{
Shiying Nan1, Yan Liang2, Yundong Qu1, Feng Liu1, Lei Wang1 and Tao Li1 \\ IDepartment of Infectious Diseases and Hepatology, The Second Hospital of Shandong University, Jinan, China \\ ${ }^{2}$ Department of Hepatology, Jinan Infectious Disease Hospital, Jinan, China
}

\begin{abstract}
Objective: To determine the frequency of liver steatosis in chronic hepatitis $\mathrm{B}(\mathrm{CHB})$ patients measured by controlled attenuation parameter (CAP) and to describe the distribution of fibrosis and inflammation in different steatosis grading. Study Design: Cross-sectional study.

Place and Duration of Study: Department of Infectious Diseases and Hepatology, The Second Hospital of Shandong University, Jinan, China, from January 2017 to December 2018.

Methodology: CHB patients who were evaluated with transient elastography (TE) for liver fibrosis and steatosis were included. Liver biochemical testing was performed within 3 days of TE measurements. Steatosis grading was assessed as S0 (no steatosis, CAP 0-247 dB/m), S1 (mild steatosis, CAP 248-267 dB/m), S2 (moderate steatosis, CAP 268-279 $\mathrm{dB} / \mathrm{m}$ ) and $\mathrm{S} 3$ (severe steatosis, CAP $>=280 \mathrm{~dB} / \mathrm{m}$ ). Statistically comparisons of continuous variables or percentages were performed.

Results: A total of 1,621 CHB patients were included. The frequencies of liver steatosis in CHB patients were $35.4 \%$ ( 574 patients), of which 314 patients (19.3\%) were diagnosed as severe steatosis (grading S3). Comparisons of age, gender and liver fibrosis in different steatosis grading revealed no statistical differences ( $p=0.109,0.075$ and 0.269 , respectively). However, ALT values in patients with severe steatosis (grading S3) were higher than those patients with steatosis grading S0 $(p<0.001)$ and S2 $(p=0.047)$.

Conclusion: Steatosis is common in CHB patients. Liver fibrosis seemed not discrepant in different steatosis grading. More obvious hepatic inflammation is seen in CHB patients with severe steatosis.
\end{abstract}

Key Words: Chronic hepatitis B, Liver steatosis, Liver fibrosis, Controlled attenuation parameter.

How to cite this article: Nan S, Liang Y, Qu Y, Liu F, Wang L, Li T. Hepatic steatosis in chronic hepatitis B as measured by controlled attenuation parameter: frequency and distribution. J Coll Physicians Surg Pak 2019; 29(10):937-41.

\section{INTRODUCTION}

Although the incidence of hepatitis B virus (HBV) infection has been greatly decreased because of the usage of hepatitis $B$ vaccine, the prevalence of HBV is still high (>5\%) in some regions of Asia and Africa, representing a relatively high amount of populations suffering from chronic hepatitis $B(\mathrm{CHB}) .{ }^{1}$ Compared to $\mathrm{CHB}$, steatosis is a more common cause of liver diseases worldwide with the improvement of living standard in modern society. ${ }^{2}$ According to the conclusion of a recent meta-analysis, the global prevalence of nonalcoholic fatty liver disease (NAFLD) is about $25.24 \% .^{3}$ For the coexistence of $\mathrm{CHB}$ and liver steatosis, a consecutive American CHB cohort including 2,734 patients found an increasing incidence of steatosis in CHB patients during 2000-2015,4 indicating the existence of more comorbidities.

Correspondence to: Tao Li, Department of Infectious Diseases and Hepatology, The Second Hospital of Shandong University,

247 Beiyuan Road, Jinan 250033, China.

E-mail:022303052@163.com

Received: March 30, 2019; Revised: June 04, 2019;

Accepted: July 09, 2019
Both $\mathrm{CHB}$ and liver steatosis are important causes for liver cirrhosis, hepatocellular carcinoma ( $\mathrm{HCC})$, and liver-related mortality. ${ }^{1,2}$ Previous studies revealed that metabolic or genetic factors may be contributed to the coexistence of $\mathrm{CHB}$ and liver steatosis. ${ }^{5}$ However, researches on the interaction between HBV infection and steatosis are still far from consensus. ${ }^{5}$ Meanwhile, the relationship of steatosis grading and fibrosis stage remains controversial in clinical practice.6-8 Studies concerning frequency and distribution of liver steatosis in $\mathrm{CHB}$ patients in the real world are still needed.

Great progress has been made in the measurement of liver steatosis in recent years. ${ }^{9-11}$ Liver biopsy is the gold standard for the identification and assessment of liver steatosis; however, the invasive feature, relatively high cost, and procedure-related risk make it difficult to apply generally in clinical practice. ${ }^{2}$ Controlled attenuation parameter (CAP) performed by transient elastography (TE) offers a comparable diagnostic accuracy to biopsy ${ }^{9}$ and has been proved to be a useful tool for the assessment of liver steatosis in chronic hepatitis B. 10,11 The noninvasive feature of CAP also makes the diagnosis of liver steatosis convenient and receptive. 
As the usage of CAP by TE has simplified the procedure for diagnosing liver steatosis, the aim of the present study was to determine the frequency of liver steatosis in $\mathrm{CHB}$ patients measured by CAP and to describe the distribution of fibrosis and inflammation in different steatosis grading.

\section{METHODOLOGY}

This cross-sectional study was performed at The Second Hospital of Shandong University, Jinan, China. CHB patients (treatment-naïve or treated by antiviral agents) who were evaluated with TE for liver fibrosis and steatosis were included. The examination periods ranged from January 2017 to December 2018. Patients co-infected with other viral hepatitis or human immunodeficiency virus, excessive alcohol intake (>40 g/day), coexist with autoimmune or metabolic liver diseases were excluded. Liver biochemical testing was performed within 3 days of TE measurements. The study protocol was approved by the Ethics Committee of The Second Hospital of Shandong University.

Measurements of CAP and liver stiffness (LS) were performed by TE with FibroScan (Echosens, Paris, France). Operations specification followed recommendations of EASL-ALEH clinical practice guidelines. ${ }^{12}$ Simply speaking, patients should be in fasting state for at least two hours and the tip of the probe transducer was placed on the skin of $9^{\text {th }}$ to $11^{\text {th }}$ costal space. Ten validated measurements with a success rate of at least $60 \%$ and an interquartile range (IQR) less than $30 \%$ of median LSM were considered as reliable. CAP was recorded as decibel per meter $(\mathrm{dB} / \mathrm{m})$ and $\mathrm{LSM}$ was recorded as kilopascal $(\mathrm{kPa})$.

Steatosis grading was assessed as SO (no steatosis, CAP 0-247 dB/m), S1 (mild steatosis, CAP 248-267 dB/m), S2 (moderate steatosis, CAP 268-279 dB/m) and S3 (severe steatosis, CAP $>=280 \mathrm{~dB} / \mathrm{m}$ ), 6,13 representing different percents of steatotic hepatocytes $(<10 \%, 10-33 \%$, $34-66 \%$ and $\geq 67 \%$, respectively). According to recommendations of EASL-ALEH clinical practice guidelines, severe liver fibrosis was defined as LS measurements more than $9.0 \mathrm{kPa}$ in $\mathrm{CHB}$ patients with normal alanine aminotransferase (ALT) values and more than $12.0 \mathrm{kPa}$ in CHB patients with elevated ALT values.6,12
Clinical data included patients' age, gender, values of ALT, CAP and LS. Virological characteristics of HBV were not recorded because of obvious decrease of hepatitis $B$ viremia under the influence of antiviral therapy.

Kolmogorov-Smirnov test was used to evaluate the normality of continuous variables. The latter were expressed as mean \pm standard deviation (SD) or median (IQR). Multiple 2-group comparisons were performed by the Kruskal-Wallis test. Nonparametric Spearman rank correlation analysis was used for correlation test between variables. The statistically significant level of p-value was set as 0.05 (two-tailed). All statistical analyses were performed using IBM SPSS Statistics Version 22.0 (IBM Corp., Armonk, NY, USA).

\section{RESULTS}

A total of 1,621 CHB patients with results of CAP and LS performed by TE were included for this cross-sectional study. The mean age of the included CHB patients was $46.5 \pm 12.1$ years (median 46.6 years) and most patients were of male gender (1119 patients, 69.0\%). The median levels of ALT, CAP and LS for included CHB patients were 27.0 (IQR 19.0-39.0) U/L, 226.0 (IQR 193.0-267.5) $\mathrm{dB} / \mathrm{m}$, and $7.9(5.7-12.0) \mathrm{kPa}$, respectively. A total of 629 patients $(38.8 \%)$ were diagnosed as severe fibrosis, according to their LS values.

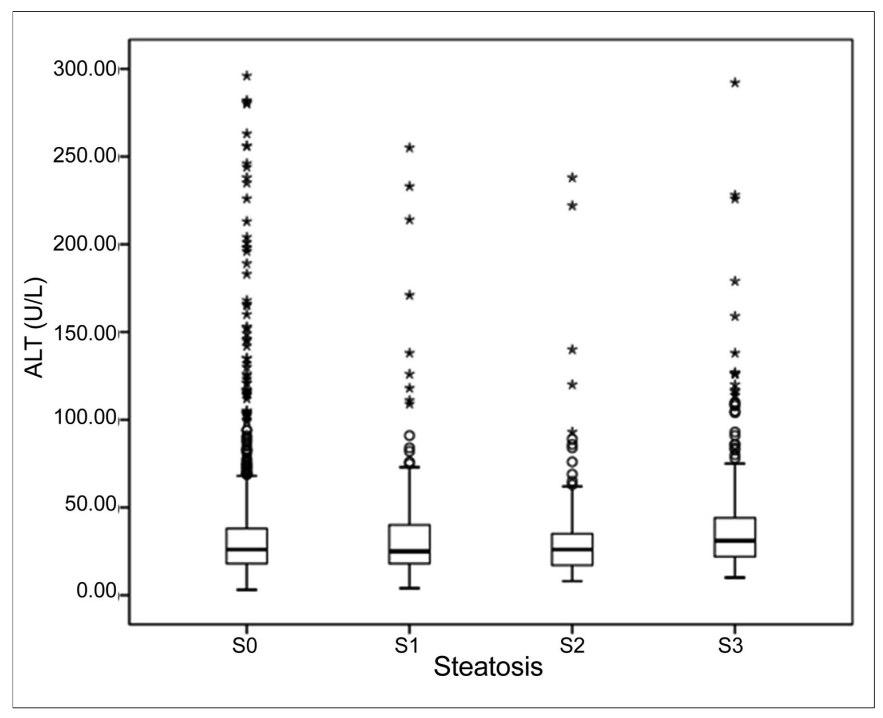

Figure 1:. Comparisons of ALT values between CHB patients with different steatosis grading.

$\mathrm{ALT}=$ Alanine aminotransferase; $\mathrm{CHB}=$ Chronic hepatitis $\mathrm{B}$.

Table I: Characteristics of CHB patients with different steatosis grading.

\begin{tabular}{|c|c|c|c|c|c|}
\hline & \multicolumn{4}{|c|}{ Steatosis grading } & \multirow[t]{2}{*}{$\mathrm{p}$-value } \\
\hline & S0 $(n=1047)$ & S1 $(n=169)$ & $S 2(n=91)$ & S3 $(n=314)$ & \\
\hline Age (years) & $46.2 \pm 12.3$ & $47.8 \pm 11.3$ & $48.4 \pm 10.8$ & $46.5 \pm 11.8$ & 0.109 \\
\hline Gender (male/female) & $708 / 339$ & $113 / 56$ & $62 / 29$ & $236 / 78$ & 0.075 \\
\hline ALT $(U / L)^{*}$ & $26.0(18.0-38.0)$ & $25.0(18.0-40.5)$ & $26.0(17.0-35.0)$ & $31.0(22.0-44.3)$ & $<0.001$ \\
\hline $\mathrm{CAP}(\mathrm{dB} / \mathrm{m})^{*}$ & $204.0(178.0-224.0)$ & $258.0(252.0-262.0)$ & $274.0(270.0-276.0)$ & $304.0(291.0-331.0)$ & $<0.001$ \\
\hline LS $(\mathrm{kPa})^{*}$ & $8.1(5.7-12.7)$ & $8.2(5.7-12.9)$ & $7.4(5.8-11.5)$ & $7.7(5.6-11.1)$ & 0.269 \\
\hline
\end{tabular}

*Median (interquartile range); \#Kruskal-Wallis test.

$\mathrm{CHB}=$ Chronic hepatitis B; $A L T=$ Alanine aminotransferase; $C A P=$ Controlled attenuation parameter; $L S=$ Liver stiffness. 
The frequency of liver steatosis in CHB patients were $35.4 \%$ (574 patients), of which 314 patients (19.3\%) were diagnosed as severe steatosis (grading S3). The characteristics of inflammation and fibrosis in patients with different steatosis grading were described in Table I. Comparisons of age, gender and liver fibrosis in different steatosis grading revealed no statistical differences $(p=0.109,0.075$ and 0.269 , respectively) (Table I). However, ALT values in patients with severe steatosis (grading S3) were higher than those in patients with steatosis grading S0 $(p<0.001)$ and $S 2(p=0.047)$, other 2-group comparisons of ALT values between different steatosis grading revealed no statistical differences (Figure 1). Similarly, the correlation between liver fibrosis and steatosis grading hinted no statistical significance $(p=-0.042, p=0.088)$, while Spearman rank analysis showed a statistical correlation for ALT values and steatosis grading $(p=0.108, p<0.001)$.

\section{DISCUSSION}

The present study described the frequency of liver steatosis in CHB patients measured by CAP and explored the distribution of fibrosis and inflammation in different steatosis grading. A frequency of $35.4 \%$ for liver steatosis in $1621 \mathrm{CHB}$ patients was explored, and a correlation of obvious hepatic inflammation (higher ALT levels) and severe steatosis (CAP $\geq 280 \mathrm{~dB} / \mathrm{m}$ ) was observed in this study. The study found no association between fibrosis stage and steatosis grading.

The present study revealed a frequency of $35.4 \%$ for liver steatosis in $\mathrm{CHB}$ patients, which was in consistent with previous reports performed by Pais et al. in European populations (21\%), ${ }^{8}$ and Seto et al. in Asian populations $(40.8 \%) .{ }^{6}$ Karacaer et al. reported a frequency of $11.4 \%$ in a retrospective study performed in Turkey. However, subjects included in this study were young male CHB patients, which should be the reason for the relatively low incidence. ${ }^{7}$ The above conclusions indicated the common occurrence for comorbidities of $\mathrm{CHB}$ and steatosis, which should arouse the attention of clinicians.

Although frequency for comorbidities of the above diseases is relatively high, the interaction between HBV and liver steatosis remains uncertain. 14-16 When hepatocytes are infected with HBV, liver injury is not caused by direct attack of the virus. The immune response induced by HBV is the main mechanism of liver inflammation and injury, in other words, the severity of liver injury depends on interactions between HBV, host immune response, and infected hepatocytes. 1,5,17 On the other hand, metabolic or genetic factors may be contributed to the susceptibility or disease progression of liver steatosis, $2,5,8$ and the incidence of liver-related death may also increased as liver steatosis progresses. 2,5 However, when the two pathogenic factors coexist, researchers are not sure whether HBV-related steatosis is caused by metabolic factors of infected individuals or by the effect of HBV on lipid metabolism pathways.5,14,18 Although liver steatosis has been proved as an additional predictor for progression of hepatic fibrosis and occurrence of HCC in patients with chronic hepatitis $\mathrm{C}(\mathrm{CHC}),{ }^{19}$ the different pathogenic mechanisms of HBV and hepatitis $C$ virus make the above findings of little referenced value.

In view of the uncertainty on interaction mechanisms between HBV and steatosis, the controversial relationship between steatosis grading and fibrosis stage is not surprising in clinical practice. The present study found no statistical differences for liver fibrosis in CHB patients without steatosis, with mild steatosis, moderate steatosis, and severe steatosis. The irrelevance between liver steatosis and fibrosis was consistent with conclusions of Pais et al.8 However, Seto et al. found that severe steatosis was associated with a high incidence of severe fibrosis. ${ }^{6}$ The constituent ratios of patients with severe fibrosis in the current study and Seto's study were $38.8 \%(629 / 1621)$ and $12.8 \%$ (178/1389), respectively. Therefore, the inconsistent conclusions may be contributed to diverse characteristics of included $\mathrm{CHB}$ patients. Be different from association between liver steatosis and fibrosis, the present study found that ALT values in patients with severe steatosis (grading S3) seemed higher than those in patients with steatosis grading SO and S2, respectively, indicating more severe liver inflammation in $\mathrm{CHB}$ patients with severe steatosis. As severe inflammation accelerates the progression of liver disease, ${ }^{1}$ severe steatosis might indicate worse outcomes in $\mathrm{CHB}$ patients. Previous cohort studies revealed that steatosis was associated with higher risk of HCC progression, ${ }^{14-16}$ which confirms the above speculation.

Influence of steatosis to HBV viral load in CHB patients also remains controversial.14,18,20 Hui et al. discovered an inverse relationship between liver steatosis and viral replication. 20 However, Zhu et al. found that hepatitis B viremia was not related to NAFLD. ${ }^{18}$ Most CHB patients included in the current study had been treated with nucleos(t)ide analogues, therefore, it was difficult to explore the relationship between steatosis and $\mathrm{HBV}$ viral load because of dramatic declining HBV DNA.

The risk of NAFLD in males has been traditionally considered higher than females. However, conclusions remain controversial.2,21 Previous studies also revealed that prevalence of NAFLD might increase with age. ${ }^{2}$ In fact, the distribution of age and gender in patients with different steatosis grading may vary because of the discrepancy in genetic factors, living habits and economic levels among different regions.2,21 The interaction of multiple factors may explain the nonrelevance between gender/age and steatosis grading in the present study. 
The main limitation of the current study should be the failure to explore relationship between steatosis and HBV viral load because of the influence of antiviral therapy. Therefore, a prospective cohort, including treatment-naïve CHB patients, has been conducted by the authors to clarify the above inconsistencies. Future longitudinal studies revealing risks of several metabolic parameters and the effect of metabolic-directed interventions for clinical outcomes are also greatly needed. 14

\section{CONCLUSION}

On the basis of the present cross-sectional study with adequate sample size, it can be concluded that steatosis is common in CHB patients. Liver fibrosis seemed not discrepant in different steatosis grading. More obvious hepatic inflammation is seen in CHB patients with severe steatosis, which might indicate worse outcomes in those patients.

\section{ETHICAL APPROVAL:}

The study protocol was approved by the Ethics Committee of The Second Hospital of Shandong University (No. KYLL2018(LW)026).

\section{PATIENTS' CONSENT:}

Informed consents have been obtained from patients to publish the data concerning this case.

\section{CONFLICT OF INTEREST:}

Authors declared no conflict of interest.

\section{AUTHORS' CONTRIBUTION:}

SN: Patients' follow-up, data collection, statistical analysis, manuscript writing and revision.

YL: Patients' follow-up, data collection.

YQ: Conception of the manuscript, study design, patients' follow-up, manuscript revision.

FL: Conception of the manuscript, study design, patients' follow-up.

LW: Study design, patients' follow-up.

TL: Conception of the manuscript, study design, patients' follow-up, data collection, statistical analysis, manuscript writing and revision.

FUNDING: This study was funded by the National Key Research and Development Program of China (No. 2017 YFC0908100, No. 2017YFC0908104) and the Project of Science and Technology Development Plan of Shandong Province (No. 2013GSF11808).

\section{REFERENCES}

1. WHO. Guidelines for the prevention, care and treatment of persons with chronic hepatitis B infection( 2015). http:// www. who.int/hepatitis/publications/hepatitis-b-guidelines/en/ (2019-03-28).
2. Chalasani N, Younossi Z, Lavine JE, Charlton M, Cusi K, Rinella $M$, et al. The diagnosis and management of nonalcoholic fatty liver disease: Practice guidance from the American Association for the Study of Liver Diseases. Hepatology 2018; 67:328-57.

3. Younossi ZM, Koenig AB, Abdelatif D, Fazel Y, Henry L, Wymer M. Global epidemiology of nonalcoholic fatty liver disease-Metaanalytic assessment of prevalence, incidence, and outcomes. Hepatology 2016; 64:73-84.

4. Liu A, Le A, Zhang J, Wong C, Wong C, Henry L, et al. Increasing comorbidities in chronic hepatitis $\mathrm{B}$ patients: Experience in primary care and referral practices during 20002015. Clin Trans/ Gastroenterol 2018; 9:141.

5. Lin CW, Huang XL, Liu HL, Wang Y. Interactions of hepatitis $B$ virus infection with nonalcoholic fatty liver disease: Possible mechanisms and clinical impact. Dig Dis Sci 2015; 60: 3513-24.

6. Seto WK, Hui RWH, Mak LY, Fung J, Cheung KS, Liu KSH, et al. Association between hepatic steatosis, measured by controlled attenuation parameter, and fibrosis burden in chronic hepatitis B. Clin Gastroenterol Hepatol 2018; 16:57583.e2.

7. Karacaer Z, Okur G, Cermik H, Altun D. Is there an influence of hepatic steatosis on fibrosis and necroinflammation in young patients with chronic viral hepatitis B? Postgrad Med 2016; 128:697-700.

8. Pais R, Rusu E, Zilisteanu D, Circiumaru A, Micu L, Voiculescu M, et al. Prevalence of steatosis and insulin resistance in patients with chronic hepatitis $B$ compared with chronic hepatitis $C$ and non-alcoholic fatty liver disease. Eur J Intern Med 2015; 26: 30-6.

9. Brener S. Transient elastography for assessment of liver fibrosis and steatosis: An evidence-based analysis. Ont Health Technol Assess Ser 2015; 15:1-45.

10. Ferraioli G, Tinelli C, Lissandrin R, Zicchetti M, Dal Bello B, Filice $\mathrm{G}$, et al. Controlled attenuation parameter for evaluating liver steatosis in chronic viral hepatitis. World J Gastroenterol 2014; 20:6626-31.

11. Xu L, Lu W, Li P, Shen F, Mi YQ, Fan JG. A comparison of hepatic steatosis index, controlled attenuation parameter and ultrasound as noninvasive diagnostic tools for steatosis in chronic hepatitis B. Dig Liver Dis 2017; 49:910-7.

12. European Association for Study of Liver, Asociacion Latinoamericana para el Estudio del Higado. EASL-ALEH Clinical Practice Guidelines: Non-invasive tests for evaluation of liver disease severity and prognosis. J Hepatol 2015; 63: 237-64.

13. Karlas T, Petroff D, Sasso M, Fan JG, Mi YQ, de Lédinghen V, et al. Individual patient data meta-analysis of controlled attenuation parameter (CAP) technology for assessing steatosis. J Hepatol 2017; 66:1022-30.

14. Seto WK. Chronic hepatitis $B$ and metabolic risk factors: A call for rigorous longitudinal studies. World J Gastroenterol 2019; 25:282-6.

15. Chen Y, Fan C, Chen Y, Liu H, Wang S, Dong P, et al. Effect of hepatic steatosis on the progression of chronic hepatitis $B$ : A prospective cohort and in vitro study. Oncotarget 2017; 8: 58601-10.

16. Lee YB, Ha Y, Chon YE, Kim MN, Lee JH, Park H, et al. Association between hepatic steatosis and the development of 
hepatocellular carcinoma in patients with chronic hepatitis B. Clin Mol Hepatol 2019;25:52-64.

17. Terrault NA, Lok ASF, McMahon BJ, Chang KM, Hwang JP, Jonas MM, et al. Update on prevention, diagnosis, and treatment of chronic hepatitis B: AASLD 2018 hepatitis B guidance. Hepatology 2018; 67:1560-99.

18. Zhu L, Jiang J, Zhai X, Baecker A, Peng $\mathrm{H}$, Qian J, et al. Hepatitis $B$ virus infection and risk of non-alcoholic fatty liver disease: A population-based cohort study. Liver Int 2019; 39:70-80.

19. Cardoso AC, Perez RM, de Figueiredo-Mendes C, Carvalho Leite N, Moraes-Coelho HS, Villela-Nogueira CA. Prevalence and predictive factors of moderate/severe liver steatosis in chronic hepatitis $\mathrm{C}(\mathrm{CHC})$ infected patients evaluated with controlled attenuation parameter (CAP). J Viral Hepat 2018; 25:1244-50.

20. Hui RWH, Seto WK, Cheung KS, Mak LY, Liu KSH, Fung J, et al. Inverse relationship between hepatic steatosis and hepatitis B viremia: Results of a large case-control study. J Viral Hepat 2018; 25:97-104.

21. Zhu JZ, Zhou QY, Wang YM, Dai YN, Zhu J, Yu CH, et al. Prevalence of fatty liver disease and the economy in China: A systematic review. World J Gastroenterol 2015; 21: 5695-706.

.......... 